\title{
Self-Medication Practices in Surrounding Communities of Birat Medical College and Teaching Hospital of Eastern Nepal
}

\author{
Surya Bahadur Parajuli, ${ }^{1,2}$ Anjali Mishra, ${ }^{1}$ Heera KC, ${ }^{3}$ Prem Bhattarai, ${ }^{4}$ Sharad Karki, ${ }^{5}$ Rajan \\ Pandit, ${ }^{2,6}$ Prasanna Dahal ${ }^{7}$ \\ ${ }^{1}$ Department of Community Medicine, Kathmandu University-Birat Medical College \& Teaching Hospital, \\ Tankisinuwari, Nepal, ${ }^{2}$ NIH-NHLBI Fellow, Translational research on CVDs in Nepal, Dhulikhel Hospital, Nepal, \\ ${ }^{3}$ Department of Nursing, Purbanchal University-Birat Health College, Biratnagar, Nepal, ${ }^{4}$ School of Health Sciences, \\ University of Tasmania, Tasmania, Australia, ${ }^{5}$ Discipline of Pharmacy, Graduate School of Health, University of \\ Technology Sydney, NSW, Australia, ${ }^{6}$ Department of Physiology, Nepal Medical College \& Teaching Hospital, \\ Kathmandu, Nepal, ${ }^{7}$ Department of Clinical Pharmacy, Purbanchal University College of Medical and Allied Health \\ Sciences, Gothgaun, Nepal.
}

\begin{abstract}
Background: Self-medication is the use of drugs to treat self-diagnosed disorders/symptoms, or the intermittent/continued use of a prescribed drug for chronic/recurrent disease/symptoms (WHO). It is the cause for antibiotic resistance, inappropriate treatment, financial burden and many deaths. WHO listed self-medication as one of the priority research area at the local context. The objective of the study was to find the prevalence and pattern of self-medication in surrounding communities of Birat Medical College and Teaching Hospital. Methods: A community-based cross-sectional study was conducted at the surrounding communities of Birat Medical College from $1^{\text {st }}$ August 2018 to $15^{\text {th }}$ December 2018. Multistage sampling was used to collect information from 348 household having family members aged 16 years and above. Ethical approval was taken from Institutional Review Committee of Birat Medical College. Pre-tested semi-structured questionnaire was used. Results: The mean age of the participants was $40.5 \pm 15.9$ years. Prevalence of self-medication was $44.04 \%$. Majority took self-medication for headache $43.6 \%$ followed by common cold $39.1 \%$ etc. Majority used allopathic drugs $82.7 \%$ followed by traditional healers $9.8 \%$. Common medication were antipyretics $18.8 \%$, antibiotics $16.5 \%$, proton pump inhibitor $7.5 \%$, antihistamines $6.8 \%$ etc. The reason behind self-medication were low cost $30.1 \%$, time saving $24.1 \%$, illness too trivial $/ \mathrm{mild}$ for consultation $18.8 \%$, quick relief $18.1 \%$, high doctor fee $15 \%$, lack of awareness $13.5 \%$, familiar with treatment options $12.8 \%$, long waiting line in hospital $12 \%$ etc. Out of them $8.3 \%$ noticed side effects of selfmedication. Out of all $59.5 \%$ felt the need of awareness program on rational use of medicines. Age, sex, marital status, ethnicity, religion, education and occupation of participants, education and occupation of head of household, poverty status, family type, house residence type, type of house has no significant association with self-medication. Participants residing in alani/rent households were 1.93 times more likely to selfmedicate than those residing in their own and participants having negative attitude were 1.90 times more likely to self-medicate than those who had positive attitude and both were statistically significant. Conclusions: The burden of self-medication was present and allopathic drugs including antibiotics were common. Adverse drug reactions were reported but participants were unaware about the place to report. Participants had negative attitude towards self-medication which is harmful for their health. As pharmacy was the common source of self-medication, the prescription based medicine dispensary should be advocated.
\end{abstract}

Keywords: AMR; hospitals; Nepal; self-medication.

\section{INTRODUCTION}

Self-medication is defined as "the use of drugs to treat self-diagnosed disorders or symptoms, or the intermittent or continued use of a prescribed drug for chronic or recurrent disease or symptoms. ${ }^{1}$ It is common in developing countries where it has both economic and social implications. ${ }^{2}$ Antibiotics are the most commonly purchased drugs worldwide with or without prescription and are essential treatments for developing world where infectious diseases are still the most common cause of death. ${ }^{3}$ One of the major cause of antibiotic resistance is self-medication. ${ }^{4}$ Various studies reported that selfmedication may lead to delay in health care seeking in appropriate place which results in paradoxical economic loss due to delay in the diagnosis of underlying conditions and actual treatment. ${ }^{5}$ The

Correspondence: Dr. Surya Bahadur Parajuli, Lecturer, Department of Community Medicine, Kathmandu UniversityBirat Medical College \& Teaching Hospital, Tankisinuwari, Morang, Nepal. Email: drsathii@yahoo.com. ORCID: https://orcid.org/0000-0003-0386-9273. DOI: 10.3126/jcmsn.v15i1.23021. Article received: 2019-01-15. Article accepted: $2019-03-02$. 
inappropriate drug use practices common in selfmedication are short duration of treatment, inadequate dose, sharing of medicines, and avoidance of treatment upon the improvement of disease symptoms. ${ }^{6}$ Different studies conducted in Nepal reported that self-medication prevalence were $59 \%$ in general population at Pokhara, ${ }^{7} 50.7 \%$ in nursing students at Chitwan, ${ }^{8} 35.1 \%$ in dental students at Kathmandu, ${ }^{9}$ and $26.2 \%$ in medical students at Pokhara. ${ }^{10}$ In a recent study at B. P. Koirala Institute of Health Sciences among the health sciences students self-prescription of paracetamol was seen among $90.1 \%{ }^{11}$

World health organization (WHO) emphasized selfmedication research in local context is one of the priority issue to reveal real scenario of selfmedication. $^{12}$ Government of Nepal nominated Department of Drug Administration (DDA) in October 2004 as the focal point (National Pharmacovigilance Center) to liaison with WHO collaborating center for International Drug Monitoring, Sweden and started collecting adverse drug reactions. Nepal became a WHO Programme member in July 2006. ${ }^{13}$ Few research suggested to strengthen the Pharmacovigilance Programme in Nepal. ${ }^{14-16}$ After promulgation of new constitution of Nepal in 20 Sept $2015,{ }^{17}$ the local governments are also responsible for federal health care system. Previous research suggested that self-medication is common and pharmacovigilance in Nepal is a propriety issue. The research on self-medication is found only on specific group of health science students. So the need to find actual status of selfmedication at community setting in eastern Nepal is the most.

With the evidence form these findings, further studies can be conducted in other community settings which may be helpful to the local government to formulate their policy on rationale use of the drugs. There is need to have their own evidence based health policy. The another implication of this research is that Birat Medical College \& Teaching Hospital can initiate to manage the growing burden of self-medication and adverse drug reactions (ADRs) to its surrounding communities through health education, policy advocacy and being a regional center for pharmacovigilance at Biratnagar of eastern Nepal. This study was conducted to find the prevalence and pattern of self-medication practices in surrounding communities of Birat Medical College and Teaching Hospital.

\section{METHODS}

This was a community-based cross sectional study conducted at the surrounding communities of Birat Medical College and Teaching Hospital from $1^{\text {st }}$ August 2018 to $15^{\text {th }}$ December 2018. Ward number
1,2 and 3 of Budhiganga Rural Municipality were taken. Multistage sampling was used to collect information from 348 households having family members aged 16 years and above. According to research done at Pokhara valley of Western Nepal in general population, the selfmedication prevalence was reported as $59 \%{ }^{7}$ By using a formula $\mathrm{N}=4 \mathrm{PQ} / \mathrm{L} 2$ with $10 \%$ permissible error sample size was $=4$ X59X41/5.9X5.9 $=$ $9676 / 34.81=277.97$ and adding non response rate of $20 \%=278+56=334$ was the minimum sample size. We took 348 participants for the study. Ethical approval was taken from Institutional Review Committee (IRC) of Birat Medical College and Teaching Hospital. Informed consent was taken from each individual who fulfilled the inclusion criteria. Pre-tested semi-structured questionnaire were delivered through ODK Collect software. House to house visit with face to face interview was done. The collected data were extracted from the ODK software in SPSS file format. Univariate and bivariate analysis were done. The findings were presented in tables and graphs. For all statistical tests, probability of significance was set at $5 \%$ level $(\mathrm{p}<0.05)$

\section{RESULTS}

In this community based cross sectional study, 348 participants were taken from surrounding communities of Birat Medical College and Teaching Hospital of Budhiganga Rural Municipality of Morang district of eastern Nepal. Table 1 shows the socio-demographic distribution of study participants. The mean age of the participants was $40.5( \pm 15.9)$ years ranged from 16-85 years. Majority of them were female 217 (62.4\%), married 287 (82.5\%), Janajati ethnicity $217(62.4 \%)$ and Hindu 339 (97.4\%). Majority were illiterate 161 (46.3\%) and $62(17.8 \%)$ had SLC and above academic qualification. Similarly majority of head of household were illiterate 209 (60.1\%). Majority of the participants were housemaker 111 $(31.9 \%)$ and head of household were daily wage worker $112(32.2 \%)$. Majority of them belonged to nuclear family $237(68.1 \%)$. Out of them $73.3 \%$ had their own land and rural residence $283(81.3 \%)$. Semi-pakka house was common 158 (45.4\%). The average family monthly income was 25000 NPR. The median walking distance to nearest health center/medical shop was 10 minutes. As in Table 2, among the 348 study participants, 302 had some sorts of illness. The cost of treatment varied from NPR 100 to NPR 300000. The medicine prescriber were listed in two groups viz. valid prescriber and self -medication user. Among those who had some sorts of illness, the prevalence of selfmedication was found to be $133(44.04 \%)$. The 
Parajuli et al. Self-Medication Practices in Surrounding Communities of Birat Medical..

\begin{tabular}{|c|c|c|}
\hline \multicolumn{3}{|c|}{$\begin{array}{l}\text { Table 1. Socio-demographic characteristics of the study } \\
\text { participants. }(n=348)\end{array}$} \\
\hline Variables & Frequency & Percent \\
\hline \multicolumn{3}{|l|}{ Age in years } \\
\hline $16-35$ & 154 & 44.3 \\
\hline $36-55$ & 120 & 34.5 \\
\hline $56-85$ & 74 & 21.3 \\
\hline Mean age in years $\pm \mathrm{SD}$ & $40.5 \pm 15.9$ & \\
\hline \multicolumn{3}{|l|}{ Sex } \\
\hline Male & 131 & 37.6 \\
\hline Female & 217 & 62.4 \\
\hline \multicolumn{3}{|l|}{ Marital status } \\
\hline Married & 287 & 82.5 \\
\hline Unmarried & 35 & 10.1 \\
\hline Widow/widower/divorcee/separated & 26 & 7.4 \\
\hline \multicolumn{3}{|l|}{ Ethnicity } \\
\hline Dalit & 44 & 12.6 \\
\hline Janajati & 217 & 62.4 \\
\hline Madhesi & 45 & 12.9 \\
\hline Muslim & 2 & 0.6 \\
\hline Brahman/Chhetri & 33 & 9.5 \\
\hline Others & 7 & 2.0 \\
\hline \multicolumn{3}{|l|}{ Religion } \\
\hline Buddhist & 5 & 1.4 \\
\hline Christian & 2 & 0.6 \\
\hline Hindu & 339 & 97.4 \\
\hline Muslim & 2 & 0.6 \\
\hline \multicolumn{3}{|l|}{ Education of participant } \\
\hline Illiterate & 161 & 46.3 \\
\hline Informal & 13 & 3.7 \\
\hline 1-5 Class & 40 & 11.5 \\
\hline 6-10 Class & 72 & 20.7 \\
\hline SLC \& Above & 62 & 17.8 \\
\hline \multicolumn{3}{|l|}{ Education of Head of household } \\
\hline Illiterate & 209 & 60.1 \\
\hline Informal & 12 & 3.4 \\
\hline 1-5 Class & 45 & 12.9 \\
\hline 6-10 Class & 54 & 15.5 \\
\hline SLC \& Above & 28 & 8.0 \\
\hline \multicolumn{3}{|l|}{ Occupation of participant } \\
\hline Agriculture & 82 & 23.6 \\
\hline Business/shopkeeper & 29 & 8.3 \\
\hline Carpenter & 8 & 2.3 \\
\hline Daily wage worker & 53 & 15.2 \\
\hline Driver & 6 & 1.7 \\
\hline Foreign Employment & 3 & 0.9 \\
\hline Government Job & 1 & 0.3 \\
\hline Health care provider & 2 & 0.6 \\
\hline Housemaker & 111 & 31.9 \\
\hline Office & 8 & 2.3 \\
\hline Student & 20 & 5.7 \\
\hline Teacher & 7 & 2.0 \\
\hline Unemployment & 18 & 5.2 \\
\hline Occupation of Head of household & & \\
\hline Agriculture & 97 & 27.9 \\
\hline Business & 43 & 12.4 \\
\hline Carpenter & 13 & 3.7 \\
\hline Daily wage worker & 112 & 32.2 \\
\hline Driver & 14 & 4.0 \\
\hline Foreign employment & 11 & 3.2 \\
\hline Housemaker & 29 & 8.3 \\
\hline Office & 25 & 7.2 \\
\hline Teacher & 4 & 1.1 \\
\hline Family type & & \\
\hline Nuclear & 237 & 68.1 \\
\hline Joint & 111 & 31.9 \\
\hline Land status & & \\
\hline Ailani & 84 & 24.1 \\
\hline Own & 255 & 73.3 \\
\hline Rent & 9 & 2.6 \\
\hline House residence type & & \\
\hline Rural & 283 & 81.3 \\
\hline Semi Urban & 63 & 18.1 \\
\hline Urban & 2 & 0.6 \\
\hline Type of house & & \\
\hline Kachha & 141 & 40.5 \\
\hline Pakka & 49 & 14.1 \\
\hline Semi pakka & 158 & 45.4 \\
\hline
\end{tabular}

\begin{tabular}{|lcc|}
\hline \multicolumn{3}{|l|}{ Table 2. Medicine prescribers. $(\mathbf{n}=\mathbf{3 0 2})$} \\
\hline Valid prescriber (169) & Frequency & Percent \\
Consultant Doctor & 117 & 69.2 \\
Medical officer & 26 & 15.4 \\
Health Assistant & 17 & 10.1 \\
CMA & 9 & 5.3 \\
Self-medication user & & \\
(n=133) & & \\
Pharmacy & 90 & 67.7 \\
Buy own self & 17 & 12.8 \\
Traditional healer & 13 & 9.8 \\
Street vendors & 6 & 4.5 \\
Family members/relatives & 4 & 3 \\
Friends & 3 & 2.3 \\
\hline
\end{tabular}

mean age at first self-medication is $28.3 \pm 14.2$ years. The pharmacy was the major source of selfmedication $90(67.7 \%)$. As in table 3, the common symptoms/disorder were headache $58(43.6 \%)$, common cold 52 (39.1\%), fever 47 (35.3\%), cough $28(21.1 \%), \%)$, muscular pain $19(14.3 \%)$, pain abdomen $16(12 \%)$, diarrhea $16(12 \%)$, common flu $12(9 \%)$, constipation $11(8.3)$, allergy 10 $(7.5 \%)$, skin problems $5(3.8 \%)$, menstrual problems $4(3 \%)$, high blood pressure $3(2.3 \%)$,

\begin{tabular}{|lcc|}
\hline $\begin{array}{l}\text { Table 3. Indications } \\
\text { *Multiple responses }\end{array}$ & for & self-medication. $(\mathbf{n}=\mathbf{1 3 3})$ \\
\hline Variables & Frequency & Percent \\
Headache & 58 & 43.6 \\
Common cold & 52 & 39.1 \\
Fever & 47 & 35.3 \\
Cough & 28 & 21.1 \\
Muscular Pain & 19 & 14.3 \\
Pain abdomen & 16 & 12.0 \\
Diarrhea & 16 & 12.0 \\
Common flu & 12 & 9.0 \\
Constipation & 11 & 8.3 \\
Allergy & 10 & 7.5 \\
Skin problems & 5 & 3.8 \\
Menstrual problems & 4 & 3.0 \\
High blood pressure & 3 & 2.3 \\
Jaundice & 1 & 0.8 \\
Asthma & 1 & 0.8 \\
Chest pain & 1 & 0.8 \\
Ear discharge & 1 & 0.8 \\
Throat problem & 1 & 0.8 \\
\hline
\end{tabular}

Jaundice $1(0.8 \%)$ etc. As in table 4, majority of the participants $110(82.7 \%)$ used allopathic drugs followed by traditional healers $13(9.8 \%)$ and ayurvedic drugs 10 (7.5\%). Antipyretics 25 $(18.8 \%)$, antibiotics $22(16.5 \%)$, proton pump inhibitors $10(7.5 \%)$ etc. are the common drugs. The most common form of medicines used was tablet $104(78.2 \%)$ followed by syrup $16(12 \%)$, powder $8(6 \%)$ and ointment $5(3.8 \%)$. Table 5 shows reason to practice self-medication. They were using self-medication because low cost 40 $(30.1 \%)$, time saving $32(24.1 \%)$, illness too trivial/mild illness for consultation $25(18.8 \%)$, quick relief $24(18.1 \%)$ etc. Table 6 shows side effects noticed after self-medication. Of them, 11 
Parajuli et al. Self-Medication Practices in Surrounding Communities of Birat Medical..

\begin{tabular}{|lcc|}
\hline \hline \multicolumn{3}{|l|}{$\begin{array}{l}\text { Table 4. Categories of drugs commonly used for self } \\
\text {-medication. (n=133) }\end{array}$} \\
\hline Types of drugs & Frequency & Percent \\
Allopathic & $\mathbf{1 1 0}$ & $\mathbf{8 2 . 7}$ \\
Antipyretics & 25 & 18.8 \\
Antibiotics & 22 & 16.5 \\
Proton Pump Inhibitor & 10 & 7.5 \\
Antihistaminic & 9 & 6.8 \\
Analgesics & 8 & 6.0 \\
Tonics/vitamins & 8 & 6.0 \\
Antitussives/ Cough syrups & 6 & 4.5 \\
Antidiarrheal & 5 & 3.8 \\
Topical corticosteroids & 5 & 3.8 \\
Medicines for mental health & 3 & 2.3 \\
problems & & \\
Medicine for non- & 3 & 2.3 \\
communicable disease & & \\
Antiemetic & 2 & 1.5 \\
Antispasmodic & 2 & 1.5 \\
Sedatives & 1 & 0.8 \\
Steroids & 1 & 0.8 \\
Ayurvedic & $\mathbf{1 0}$ & $\mathbf{7 . 5}$ \\
Traditional healers & $\mathbf{1 3}$ & $\mathbf{9 . 8}$ \\
\hline
\end{tabular}

Table 5. Reason to practice self-medication. $(n=133)$ * Multiple responses

\begin{tabular}{lcc|}
\hline Variables & Frequency & Percent \\
Low cost & 40 & 30.1 \\
Time saving & 32 & 24.1 \\
Illness too trivial/Mild illness for consulta- & 25 & 18.8 \\
tion & 24 & 18.1 \\
Quick relief & 20 & 15.0 \\
High doctor fee & 18 & 13.5 \\
Lack of awareness & 17 & 12.8 \\
Familiar with treatment options & 16 & 12.0 \\
Long waiting line in hospital & 13 & 9.8 \\
Belief of traditional healer & 10 & 7.5 \\
Old prescription for same illness & 10 & 7.5 \\
No doctors nearby & 9 & 6.8 \\
Thought pharmacists are also doctors & 8 & 6.0 \\
Fear of investigation at hospital/health & & \\
center & 7 & 5.3 \\
Privacy & 6 & 4.5 \\
Internet search & 4 & 3.0 \\
Fear of not being able to take medicine & & \\
regularly & 3 & 2.3 \\
Long process at hospital/health center & 2 & 1.5 \\
Avoid crowed at OPD & 2 & 1.5 \\
Academic knowledge & 2 & 1.5 \\
Rude behaviour of Health care providers & 1 & 0.8 \\
Drug advertisement & & \\
\hline
\end{tabular}

$(8.3 \%)$ reported some sorts of side effects. The common side effects were allergy 2 (18.2\%), diarrhea $2(18.2 \%)$ etc. Majority of them 5 (45.4\%) consults with pharmacist followed by stop taking medicine $3(27.3 \%)$ etc. Figure 1 shows reason for avoiding self-medication. Among the study participants having some sorts of illness 169 $(55.96 \%)$ consulted. They are avoiding selfmedication because of risk of using wrong medicine $91(53.9 \%)$, risk of adverse drug reaction $42(24.9 \%)$ and risk of wrong diagnosis 36 $(21.2 \%)$. Table 7 shows attitude of participants towards self-medication. Majority had neutral attitude 179 (51.4) followed by positive attitude 116

\begin{tabular}{|lcc|}
\hline \multicolumn{3}{|l|}{$\begin{array}{l}\text { Table 6. } \\
\text { tion taken. }(\mathbf{n}=\mathbf{1 3 3})\end{array}$} \\
\hline Variables & Frequency & Percent \\
Yes & 11 & 8.3 \\
Allergy & 2 & 18.2 \\
Diarrhea & 2 & 18.2 \\
Headache & 2 & 18.2 \\
Vomiting & 2 & 18.2 \\
Weakness & 1 & 9.1 \\
Nausea & 1 & 9.1 \\
No & 122 & 91.7 \\
Action taken after having side effects $(\mathbf{n}=11)$ \\
$\begin{array}{l}\text { Consult with } \\
\text { pharmacist }\end{array}$ & 5 & 45.4 \\
$\begin{array}{l}\text { Stop taking } \\
\text { medicine }\end{array}$ & 3 & 27.3 \\
$\begin{array}{l}\text { Switch to an- } \\
\text { other medicine }\end{array}$ & 3 & 27.3 \\
\hline
\end{tabular}

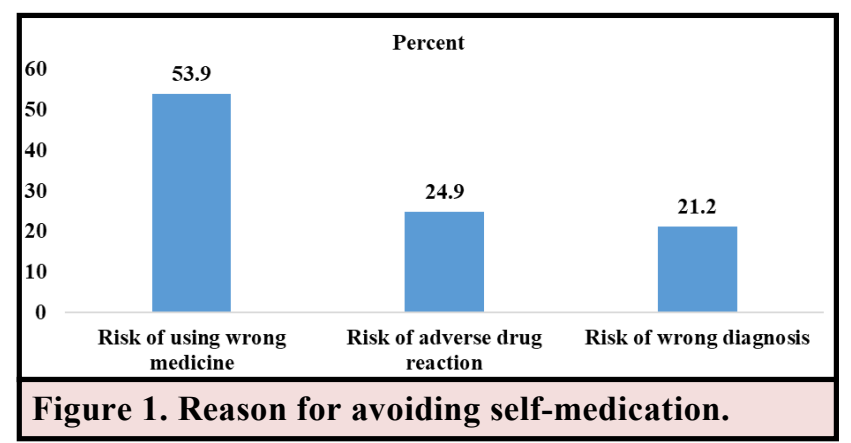

\begin{tabular}{|c|c|c|}
\hline Variables & Frequency & Percent \\
\hline Neutral Attitude & 179 & 51.4 \\
\hline Positive attitude & 116 & 33.4 \\
\hline Negative Attitude & 53 & 15.2 \\
\hline
\end{tabular}

$(33.4 \%)$ etc. Figure 2 shows need of awareness program on self-medication or rational use of medicine. Majority 207 (59.5\%) need awareness

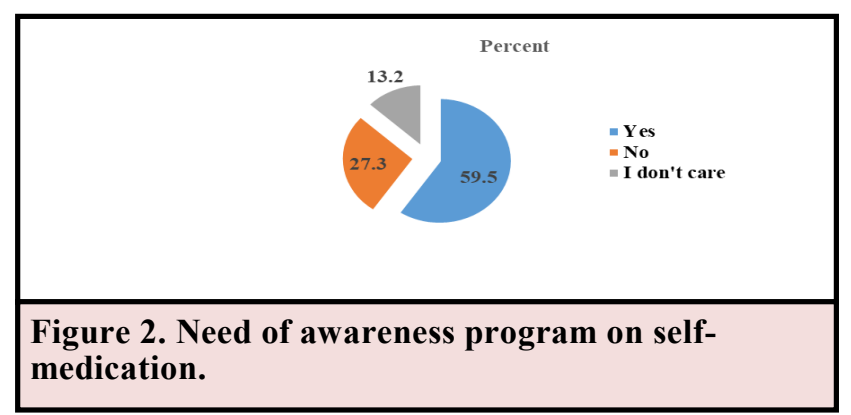

program. The association of different variables with self-medication were analysed. The bivariate analysis showed in table 8. Age, sex, marital status, ethnicity, religion, education and occupation of participants, education and occupation of head of household, poverty status, family type, house residence type, type of house, need awareness program has no significant association with self-medication. The status of land has significant association with selfmedication. The participants residing in alani/rent 
Parajuli et al. Self-Medication Practices in Surrounding Communities of Birat Medical..

\begin{tabular}{|c|c|c|c|c|c|c|}
\hline \multicolumn{7}{|c|}{$\begin{array}{l}\text { Table } 8 \text {. Association of self-medication with differ- } \\
\text { ent variables. }\end{array}$} \\
\hline \multirow[t]{2}{*}{ Variables } & \multirow[t]{2}{*}{$\begin{array}{l}\text { Catego- } \\
\text { ries }\end{array}$} & \multicolumn{2}{|c|}{$\begin{array}{c}\text { History of self- } \\
\text { medication }\end{array}$} & \multirow[t]{2}{*}{$\overline{\mathbf{O R}}$} & \multirow[t]{2}{*}{$\overline{C I}$} & \multirow[t]{2}{*}{$\bar{p}$} \\
\hline & & Yes & No & & & \\
\hline \multirow[t]{2}{*}{ Age } & $16-35 \mathrm{yrs}$ & $\begin{array}{c}53 \\
(42.7 \%)\end{array}$ & $\begin{array}{c}71 \\
(57.3 \%)\end{array}$ & \multirow[t]{2}{*}{0.9} & $\begin{array}{l}0.6 \\
- \\
1.5\end{array}$ & \multirow[t]{2}{*}{0.7} \\
\hline & $\geq 36 \mathrm{yrs}$ & $\begin{array}{c}80 \\
(44.9 \%)\end{array}$ & $\begin{array}{c}98 \\
(55.1 \%)\end{array}$ & & & \\
\hline \multirow[t]{2}{*}{ Sex } & Female & $\begin{array}{c}88 \\
(46.8 \%)\end{array}$ & $\begin{array}{c}100 \\
(53.2 \%)\end{array}$ & \multirow[t]{2}{*}{1.4} & $\begin{array}{l}0.8 \\
-\end{array}$ & \multirow[t]{2}{*}{0.2} \\
\hline & Male & $\begin{array}{c}45 \\
(39.5 \%)\end{array}$ & $\begin{array}{c}69 \\
(60.5 \%)\end{array}$ & & 0.2 & \\
\hline \multirow[t]{2}{*}{ Marital status } & Married & $\begin{array}{c}109 \\
(43.8 \%)\end{array}$ & $\begin{array}{c}140 \\
(56.2 \%)\end{array}$ & \multirow[t]{2}{*}{0.9} & $\begin{array}{l}0.5 \\
-\end{array}$ & \multirow[t]{2}{*}{0.8} \\
\hline & $\begin{array}{l}\text { Unmar- } \\
\text { ried }\end{array}$ & $\begin{array}{c}24 \\
(45.3 \%)\end{array}$ & $\begin{array}{c}29 \\
(54.7 \%)\end{array}$ & & 1.7 & \\
\hline \multirow[t]{2}{*}{ Ethnicity } & Janajati & $\begin{array}{c}90 \\
(48.45)\end{array}$ & $\begin{array}{c}96 \\
(51.6 \%)\end{array}$ & \multirow[t]{2}{*}{1.6} & $\begin{array}{l}1- \\
2.6\end{array}$ & \multirow[t]{2}{*}{0.5} \\
\hline & Other & $\begin{array}{c}43 \\
(37.1 \%)\end{array}$ & $\begin{array}{c}73 \\
(62.9 \%)\end{array}$ & & & \\
\hline \multirow[t]{2}{*}{ Religion } & Hindu & $\begin{array}{c}128 \\
(43.7 \%)\end{array}$ & $\begin{array}{c}165 \\
(56.3 \%)\end{array}$ & \multirow[t]{2}{*}{0.6} & $\begin{array}{c}0.2 \\
-\end{array}$ & \multirow[t]{2}{*}{0.5} \\
\hline & Other & $\begin{array}{c}5 \\
(55.6 \%)\end{array}$ & $\begin{array}{c}4 \\
(44.4 \%)\end{array}$ & & 2.4 & \\
\hline \multirow{2}{*}{$\begin{array}{l}\text { Education of } \\
\text { the partici- } \\
\text { pants }\end{array}$} & Illiterate & $\begin{array}{c}59 \\
(41.5 \%)\end{array}$ & $\begin{array}{c}83 \\
(58.55)\end{array}$ & \multirow[t]{2}{*}{0.8} & $\begin{array}{c}0.5 \\
-\end{array}$ & \multirow[t]{2}{*}{0.4} \\
\hline & Literate & $\begin{array}{c}74 \\
(46.3 \%)\end{array}$ & $\begin{array}{c}86 \\
(53.8 \%)\end{array}$ & & 1.3 & \\
\hline \multirow{2}{*}{$\begin{array}{l}\text { Education of } \\
\text { the head of } \\
\text { household }\end{array}$} & Illiterate & $\begin{array}{c}81 \\
(47.1 \%)\end{array}$ & $\begin{array}{c}91 \\
(52.9 \%)\end{array}$ & \multirow[t]{2}{*}{1.3} & 0.8 & \multirow[t]{2}{*}{0.2} \\
\hline & Literate & $\begin{array}{c}52 \\
(40 \%)\end{array}$ & $\begin{array}{c}78 \\
(60 \%)\end{array}$ & & 2.1 & \\
\hline $\begin{array}{l}\text { Occupation of } \\
\text { the partici- }\end{array}$ & $\begin{array}{l}\text { Profes- } \\
\text { sional }\end{array}$ & $\begin{array}{c}22 \\
(45.8 \%)\end{array}$ & $\begin{array}{c}26 \\
(54.2 \%)\end{array}$ & 1.1 & $\begin{array}{l}0.6 \\
-2\end{array}$ & 0.9 \\
\hline & $\begin{array}{l}\text { Non } \\
\text { profes- } \\
\text { sional }\end{array}$ & $\begin{array}{c}111 \\
(43.7 \%)\end{array}$ & $\begin{array}{c}143 \\
(56.3 \%)\end{array}$ & & & \\
\hline $\begin{array}{l}\text { Occupation of } \\
\text { the head of }\end{array}$ & $\begin{array}{l}\text { Profes- } \\
\text { sional }\end{array}$ & $\begin{array}{c}39 \\
(45.9 \%)\end{array}$ & $\begin{array}{c}46 \\
(54.1 \%)\end{array}$ & 1.1 & $\begin{array}{c}0.7 \\
-\end{array}$ & 0.7 \\
\hline household & $\begin{array}{l}\text { Non } \\
\text { profes- } \\
\text { sional }\end{array}$ & $\begin{array}{c}94 \\
(43.3 \%)\end{array}$ & $\begin{array}{c}123 \\
(56.7 \%)\end{array}$ & & 1.8 & \\
\hline$\underset{\text { (18) }}{\text { Poverty status }}$ & Above & $\begin{array}{c}62 \\
(48.1 \%)\end{array}$ & $\begin{array}{c}67 \\
(51.9 \%)\end{array}$ & 1.3 & 0.8 & 0.2 \\
\hline & Below & $\begin{array}{c}71 \\
(41 \%)\end{array}$ & $\begin{array}{c}102 \\
(59 \%)\end{array}$ & & 2.1 & \\
\hline Family type & & $\begin{array}{c}40 \\
(41.2 \%)\end{array}$ & $\begin{array}{c}57 \\
(58.8 \%)\end{array}$ & 0.8 & 0.5 & 0.5 \\
\hline & Nuclear & $\begin{array}{c}93 \\
(45.4 \%)\end{array}$ & $\begin{array}{c}112 \\
(54.6 \%)\end{array}$ & & 1.4 & \\
\hline Status of land & $\begin{array}{l}\text { Rent/ } \\
\text { Alani }\end{array}$ & $\begin{array}{c}38 \\
(56.7 \%)\end{array}$ & $\begin{array}{c}29 \\
(43.3 \%)\end{array}$ & $\begin{array}{c}1.9 \\
3\end{array}$ & $\begin{array}{c}1.1 \\
-\end{array}$ & $\begin{array}{l}0.0 \\
2 *\end{array}$ \\
\hline & Own & $\begin{array}{c}95 \\
(40.4 \%)\end{array}$ & $\begin{array}{c}140 \\
(59.6 \%)\end{array}$ & & 3.3 & \\
\hline $\begin{array}{l}\text { House resi- } \\
\text { dence type }\end{array}$ & Rural & $\begin{array}{c}108 \\
(43.7 \%)\end{array}$ & $\begin{array}{c}139 \\
(56.3 \%)\end{array}$ & 0.9 & $\begin{array}{c}0.5 \\
-\end{array}$ & 0.9 \\
\hline & $\begin{array}{l}\text { Semi } \\
\text { Urban }\end{array}$ & $\begin{array}{c}25 \\
(45.5 \%)\end{array}$ & $\begin{array}{c}30 \\
(54.5 \%)\end{array}$ & & 1.7 & \\
\hline Type of house & Kachha & $\begin{array}{c}56 \\
(47.9 \%)\end{array}$ & $\begin{array}{c}61 \\
(52.1 \%)\end{array}$ & 1.3 & $\begin{array}{c}0.8 \\
-\end{array}$ & 0.3 \\
\hline & $\begin{array}{l}\text { Semi/ } \\
\text { pakka }\end{array}$ & $\begin{array}{c}77 \\
(41.6 \%)\end{array}$ & $\begin{array}{c}108 \\
(58.4 \%)\end{array}$ & & 2.1 & \\
\hline $\begin{array}{l}\text { Attitude } \\
\text { towards self- } \\
\text { medication }\end{array}$ & $\begin{array}{l}\text { Negative/ } \\
\text { Neutral }\end{array}$ & $\begin{array}{c}98 \\
(49.2 \%)\end{array}$ & $\begin{array}{c}101 \\
(50.8 \%)\end{array}$ & 1.9 & $\begin{array}{l}1.2 \\
- \\
3.1\end{array}$ & $\begin{array}{l}0.0 \\
1 *\end{array}$ \\
\hline & Positive & $\begin{array}{c}35 \\
(34 \%)\end{array}$ & $68(66 \%)$ & & & \\
\hline $\begin{array}{l}\text { Need aware- } \\
\text { ness program }\end{array}$ & Yes & $\begin{array}{c}82 \\
(44.3 \%)\end{array}$ & $\begin{array}{c}103 \\
(55.7 \%)\end{array}$ & 1 & $\begin{array}{c}0.6 \\
-\end{array}$ & 0.9 \\
\hline & $\begin{array}{l}\text { No/I don't } \\
\text { care }\end{array}$ & $\begin{array}{c}51 \\
(43.6 \%)\end{array}$ & $\begin{array}{c}66 \\
(56.4 \%)\end{array}$ & & 1.6 & \\
\hline
\end{tabular}

household had 1.93 times more use of selfmedication than those who resides in own household. The attitude towards self-medication has significant association with self-medication practice. The participants having negative attitude has 1.9 times more use of self-medication than those who had positive attitude.

\section{DISCUSSION}

In this community based cross sectional study 348 participants were taken from surrounding communities of Birat Medical College and Teaching Hospital of Budhiganga Rural Municipality of Morang district of eastern Nepal. In this study the median walking distance to nearest health center/medical shop was 10 minutes which is similar to another research at Pokhara. $^{7}$ Selfmedication is common in developing countries where it has both economic and social implications. ${ }^{2}$ Antibiotics are the most commonly purchased drugs worldwide with or without prescription and are essential treatments for developing world where infectious diseases are still the most common cause of death. ${ }^{3}$ One of the major cause of antibiotic resistance is self-medication. ${ }^{4}$ The medicine prescriber were listed in two groups viz. valid prescriber and self-medication user. Among 348 study participants, 302 had some sorts of illness and among those who had some sorts of illness, the prevalence of self-medication was found to be $44.04 \%$. The mean age at first self-medication is $28.3 \pm 14.2$ years. Different studies conducted in Nepal reported that self-medication prevalence were $59 \%$ in general population at Pokhara, ${ }^{7} 50.7 \%$ in nursing students at Chitwan, ${ }^{8} 35.1 \%$ in dental students at Kathmandu, ${ }^{9} 26.2 \%$ in medical students at Pokhara, ${ }^{10} 90.1 \%$ in health sciences students at at B. P. Koirala Institute of Health Sciences for paracetamol $^{11}$ and $82 \%$ among medical students of western Nepal. ${ }^{19}$ Studies from India reported that $11.9 \%$ at urban Pondicherry, ${ }^{5} 31.3 \%$ at urban Delhi ${ }^{20}$ and $57.05 \%$ among medical students at West-Bengal. ${ }^{21}$ Studies from Sri Lanka also reported $12.2 \%$ and $7.9 \%$ prevalence of selfmedication to allopathic drugs from urban and rural areas. ${ }^{22}$

In this study, pharmacy was the major source $(67.7 \%)$ of self-medication. Study from India with similar socio-demographic characteristics found that pharmacists and pharmacy attendants play an important role in fostering self-medication among the public. ${ }^{23}$ Telling the symptoms to pharmacist $(38.1 \%)$ was the commonest method adopted to procure drugs by the users. ${ }^{5}$ The medical shop was the commonest source of medicines. ${ }^{24}$ Of them $65 \%$ of respondentsused OTC medicines because the pharmacy was near fromtheir homes. ${ }^{25}$

In this study, the common symptoms/disorder for self-medication were headache $(43.6 \%)$, common cold $(39.1 \%)$, fever $(35.3 \%)$, cough $(21.1 \%), \%)$, muscular pain $(14.3 \%)$, pain abdomen $(12 \%)$, diarrhea $(12 \%)$, common flu $(9 \%)$, constipation (8.3), allergy $(7.5 \%)$, skin problems $(3.8 \%)$, menstrual problems $(3 \%)$, high blood pressure $(2.3 \%)$, Jaundice $(0.8 \%)$ etc. Similar finding reported from other studies where headache and 
Parajuli et al. Self-Medication Practices in Surrounding Communities of Birat Medical..

fever $(60 \%){ }^{7}$ common cold $(53.3 \%),{ }^{26}$ fever $(92 \%),{ }^{25}$ fever $(31 \%)$, headache $(19 \%)$, and abdominal pain $(16.7 \%)$ were most common illnesses for self-medication. ${ }^{5}$ Even though prevalence of self-medication tends to vary in different studies, determinants and patterns of selfmedication is similar.

In this study drugs used for self-medication were allopathic drugs $(82.7 \%)$ followed by traditional healers $(9.8 \%)$ and Ayurvedic $(7.5 \%)$. Antipyretics $(18.8 \%)$, antibiotics (16.5\%), proton pump inhibitors $(7.5 \%)$ etc. were the common drugs. Similar findings reported in studies where paracetamol and antimicrobials, ${ }^{7}$ paracetamol $41.3 \%{ }^{24}$ antibiotics $38.4 \%{ }^{27}$ and NSAIDs $(33.33 \%)$, antibiotics $(10.32 \%)$, vitamins $(14.08 \%)$ and Gastro intestinal tract ailment drugs (13.61\%) are most commonly used. ${ }^{28}$

In this study major reasons for self-medication were low cost $(30.1 \%)$, time saving (24.1\%), Illness too trivial/mild illness for consultation $(18.8 \%)$, quick relief $(18.1 \%)$ etc. Similar findings were reported as mild illness, previous experience of treating a similar illness, non-availability of health personnel ${ }^{7}$ and were simple illness, previous experience of treating a similar illness and medicinal herbs were easily available in the courtyard of the house. ${ }^{24}$ In the rational use of drug workshops held on 1997-1999 in different parts of Nepal, similar factors were reported for selfmedication and are still relevant in present context of Nepal. ${ }^{29}$

In this study $55.96 \%$ consulted for their illness. They are avoiding self-medication because of risk of using wrong medicine $53.9 \%$, risk of adverse drug reaction $24.9 \%$, risk of wrong diagnosis $21.2 \%$. Among those who did self-medication, $8.3 \%$ reported some sorts of side effects. The common side effects were allergy (18.2\%) and diarrhea $(18.2 \%)$ etc. In a study at a tertiary center of New Delhi India, the most frequently seen ADRs were diarrhea, gastritis, abdominal pain followed by nausea and vomiting even in hospital setting. ${ }^{30}$ In a study from Nepal Medical College Teaching Hospital, Kathmandu, ADR is commonly reported in their pharmacovigilance center. ${ }^{31}$

In this study $59.5 \%$ felt the need of awareness program on rational use of medicine but majority had negative or neutral attitude $(66.6 \%)$ which is similar study from Pondicherry, India. ${ }^{5}$

In this study different socio-demographic variables has no significant association with self-medication which signifies that this is a common problem in all age, sex, education level, occupation status and any family type etc. The status of land has significant association with self-medication. The participants residing in alani/rent household had 1.93 times more use of self-medication than those who resides in own household. The attitude towards self-medication has significant association with self -medication practice. The participants having negative attitude had 1.9 times more use of selfmedication than those who had positive attitude. This means people who were more careless are prone to self-medication.

Government of Nepal nominated Department of Drug Administration (DDA) in October 2004 as the focal point (National Pharmacovigilance Center) to liaison with WHO collaborating center for International Drug Monitoring, Sweden and started collecting adverse drug reactions. Nepal became a WHO Programme member in July $2006{ }^{13}$ Few research suggested that time has come to strengthening the Pharmacovigilance Programme in Nepal. ${ }^{14-16,32}$ Nepal is determined to strengthen its pharmacovigilance activities. . $^{13,33}$

After promulgation of new constitution of Nepal in 20 Sept $2015,{ }^{17}$ the local government should be more responsible for self-medication issues and ADR reporting to the pharmacovigilance centres. DDA suggested to report ADR through ADR reporting form. ${ }^{34}$

\section{CONSLUSION}

The burden of self-medication was present in surrounding communities of Birat Medical College \& Teaching Hospital. Allopathic drugs including antibiotics were the common medicines used for self-medication. Adverse drug reactions were reported but participants were unaware the place to report. Participants had negative attitude towards self-medication which is harmful for their health. As pharmacy was the common source of selfmedication, the prescription based medicine dispensary should be advocated.

\section{Recommendations}

This evidence may be helpful for the local government to formulate their policy on rationale use of the drugs.Birat Medical College \& Teaching Hospital can initiate to manage the growing burden of self-medication and adverse drug reactions (ADRs) to its surrounding communities through health education, policy advocacy and being a regional center for pharmacovigilance at Biratnagar of eastern Nepal. This type of research work in other local bodies can be done. We recommend to conduct research on effect of an educational intervention on knowledge, attitude and practice of self-medication in the local bodies.

Limitation of the study

The consumption of different medication could not 
be confirmed in all the study participants as the prescription or cover of medication was not available.

\section{ACKNOWLEDGEMENTS}

The authors would like to thank all the study participants, local health centers and concerned authorities for their support. The authors would like to thank Samir Dahal and Hom Parajuli for their contribution.

Conflict of interest: We declare no conflict of interest.

\section{REFERENCES}

1. WHO. Guidelines for the regularoty assessment of medicinal products for self medication. 2000. Cited: 4 Jan 2019. Available from: http:// apps.who.int/medicinedocs/pdf/s2218e/ s2218e.pdf.

2. Parulekar M, Mekoth N, Ramesh CM, Parulekar A. Self-medication in Developing Countries: A Systematic Review. Journal of Pharmaceutical Technology, Research and Management.2016;4 (2) pp. 103-127.doi: 10.15415/jptrm.2016.42007.

3. WHO. Tackling antimicrobial resistance (AMR) together. Working paper 5.0: Enhancing the focus on gender and equity. Geneva: World Health Organization; 2018 (WHO/HWSI/AMR/2018.3). Cited: 4 Jan 2019 Available from: https:// www.who.int/antimicrobial-resistance/nationalaction-plans/AMRGenderEquityGuidanceSept2018.pdf.

4. Nepal G. Bhatta S. Self-medication with Antibiotics in WHO Southeast Asian Region: A Systematic Review.Cureus. 2018; 10(4): e2428. doi: 10.7759 /cureus. 2428 .

5. Selvaraj K, Kumar SG, Ramalingam A. Prevalence of self-medication practices and its associated factors in Urban Puducherry, India. Perspectives in clinical research. 2014;5(1):326.doi: 10.4103/2229-3485.124569.

6. Skliros E, Merkouris P, Papazafiropoulou A, Gikas A, Matzouranis G, Papafragos C, Tsakanikas I et al. Self-medication with antibiotics in rural population in Greece: a crosssectional multicenter study. BMC FamPract. 2010;8 (11):58. doi: 10.1186/14712296-11-58.

7. Shankar PR, Partha P, Shenoy N. Self-medication and non-doctor prescription practices in Pokhara valley, Western Nepal: a questionnaire-based study. BMC family practice. 2002;3:17. doi: 10.1186/1471-2296-3-17.

8. Sah AK, Jha RK, Shah DK. Self-medication with antibiotics among nursing students of Nepal. International Journal of Pharma Sciences and Research. 2016;7:427-430.

9. Pant N, Sagtani RA, Pradhan M, Bhattarai A, Sagtani A. Self-medication with antibiotics among dental students of Kathmandu prevalence and practice. Nepal Med Coll J 2015; 17(1-2): 47-53.

10.Banerjee I, Sathian B, Gupta RK, Amarendra A, Roy B, Bakthavatchalam P et al. Self-medication practice among preclinical university students in a medical school from the city of Pokhara, Nepal.
Nepal J Epidemiol. 2016;6(2); 574-581.

11. Yadav AK, Rai BK, Budhathoki SS, Ghimire A, Shrestha SR, Malla GB. Self-prescription of Paracetamol by Undergraduate Students in BP Koirala Institution of Health Sciences. Journal of the Nepal Medical Association. 2016;55(203):115. PubMed PMID: 27935916. Epub 2016/12/10. eng.

12. WHO. Priority research topics. Cited: 4 Jan 2019. Available from: http://www.who.int/ drugresistance/informationsharing/en/ priorityresearchtopics.pdf?ua $=1$.

13.Pharmacovigilance. Department of Drug Administration. Cited: 5 Jan 2019. Available from: http://www.dda.gov.np/content/ pharmacovigilance.

14.KC S, Tragulpiankit P, Gorsanam P, Edwards I, Alam K. Strengthening the Pharmacovigilance Programme in Nepal. Nepal Journal of Epidemiology.2013;3(1):230-5. doi: https:// doi.org/10.3126/nje.v3i1.8286

15.Palaian S, Izham M, Ibrahim M, Mishra P, Alam A, Ravi Shankar P et al. Pharmacovigilance Activities in Nepal. Drug-Safety (2010) 33: 889. https://doi.org/10.2165/11532480-00000000000000 .

16. Khanal S, Gyawali S, Kanodia R, Rathor RS. Pharmacovigilance: Urgent need in midwestern region of Nepal.Kathmandu University Medical Journal.2009; 7 (4) 470 . doi: https:// doi.org/10.3126/kumj.v7i4.2776.

17.List of Local Level Power. Cited: 4 Jan 2019. Available from: http:// www.lawcommission.gov.np/en/archives/782.

18. World Bank. Global Poverty Line Update. Cited: 4 Jan 2019. Available from: http:// www.worldbank.org/en/topic/poverty/brief/global -poverty-line-faq.

19. Gyawali S, Shankar PR, Poudel PP, Saha A. Knowledge, attitude and practice of selfmedication among basic science undergraduate medical students in a medical school in western Nepal. Journal of clinical and diagnostic research. 2015;9(12):FC17-22.

doi:

$10.7860 /$ JCDR/2015/16553.6988.

20.Lal V, Goswami A, Anand K. Self-medication among residents of urban resettlement colony, New Delhi. Indian journal of public health. 2007;51(4):249-51. PubMed PMID: 18232170. Epub 2008/02/01. eng.

21.Banerjee I, Bhadury T. Self-medication practice among undergraduate medical students in a tertiary care medical college, West Bengal. 
Parajuli et al. Self-Medication Practices in Surrounding Communities of Birat Medical..

Journal of postgraduate medicine. 2012;58(2):12731. PubMed PMID: 22718057. Epub 2012/06/22. eng.

22. Wijesinghe PR, Jayakody RL, de A Seneviratne R. Prevalence and predictors of self-medication in a selected urban and rural district of Sri Lanka. WHO South East Asia J Public Health. 2012;1(1):28-41. doi: 10.4103/2224-3151.206911.

23.Kamat VR, Nichter M. Pharmacies, self-medication and pharmaceutical marketing in Bombay, India. SocSci Med. 1998;47(6):779-94.PubMed PMID: 9690824. Epub 1998/08/05. eng.

24. Shankar PR, Kumar P, Theodore AM, Partha P, Shenoy N. A survey of drug use patterns in western Nepal. Singapore medical journal. 2003;44(7):3526. PubMed PMID: 14620727. Epub 2003/11/19. eng.

25.Maharjan M, Adhikari K. Practices on over the counter medication among mothers of under-five aged children attending child OPD at friends of ShantaBhawan, Boudha, Kathmandu. Health Prospect. 2018;10:19-1.doi: https://doi.org/10.3126/ hprospect.v10i0.5640

26.Sarraf DP, Karna G, Dhungana P, Lammichhane S, Rauniar GP. Pattern of Self-medication in Undergraduate Students at BP Koirala Institute of Health Sciences. Kathmandu University Medical Journal. 2017;15(57):14-8. PubMed PMID: 29446356. Epub 2018/02/16. eng.

27.Ajayi I, Omotoye O, Ajite K, Fadamiro C, Ajayi E. Self-medication practices among patients seen in a Suburban Tertiary eye care centre in Nigeria. Asian Journal of Medical Sciences.2013;5(2). doi: https:// doi.org/10.3126/ajms.v5i2.8452

28. Keche Y, Yegnanarayan R, Bhoyar S, Agrawal R, Chavan R, Mahendrakar P. Self medication pattern in rural areas in Pune, India. International Journal of Medicine and Public Health.2012;2(4). doi:10.5530/ijmedph.2.4.2

29.Joshi MP, Onta S, Khakurel B, Bhattarai K. Rational drug use workshops for consumers in Nepal. Journal of the Institute of Medicine 1999; 21: $1-81$.

30.Shah R, Karki S, Parajuli SB, Bhattarai P, Chowdhary P. Pharmacovigilance by World Health Organisation Uppsala Monitoring Center Causality Assessment Algorithm in Medicine Ward of Tertiary Care Hospital of New Delhi. Birat Journal of Health Sciences.2017;1(1):61-4. doi: https://doi.org/10.3126/bjhs.v1i1.17102.

31.Bista D, Shrestha B, Rai P, Jauhari AC, Kulshrestha V. Pattern of adverse drug reactions reported to the regional Pharmacovigilance center at Nepal Medical College and Teaching Hospital, Kathmandu. Journal of Nepal Pharmaceutical Association. 2012. 26(1), 54-61. doi: https:// doi.org/10.3126/jnpa.v26i1.6633.

32.WHO Programme for International Drug Monitoring. Cited: 5 Jan 2019. Available from: https://www.who-umc.org/globalpharmacovigilance/who-programme/

33. Uppsala Monitoring Center. Sharing knowledge to boost the Nepalese pharmacovigilance programme. July 2017.Issue 76. Cited: 5 Jan 2019. Available from: https://www.who-umc.org/ media/3271/uppsala-reports-76_web.pdf.

34.Department of Drug Administration. Adverse Drug Reactions Reporting Form. Cited: 5 Jan 2019. Available from: http://www.dda.gov.np/ download/adr\%20reporting\%20form.pdf.

Citation: Parajuli SB, Mishra A, KC H, Bhattarai P, Karki S, Pandit R, Dahal P. Self-Medication Practices in Surrounding Communities of Birat Medical College and Teaching Hospital of Eastern Nepal. JCMS Nepal. 2019;15(1):45-52. 